\title{
Contextual factors affecting health information system strengthening
}

\author{
James C. Thomas (iD ${ }^{a, b}$ \\ ${ }^{a}$ Department of Epidemiology, Gillings School of Global Public Health, University of North Carolina, Chapel \\ Hill, NC, USA; ' ${ }^{\mathrm{b}}$ MEASURE Evaluation Project, Carolina Population Center, University of North Carolina, Chapel \\ Hill, NC, USA
}

\begin{abstract}
At the turn of the century, several major efforts were initiated to combat HIV/AIDS and other major epidemics affecting low- and middle-income countries (LMICs). They were accompanied by initiatives to enable recipient countries to collect and use data to guide their public health programmes. These health information systems (HIS) typify systems in that they have multiple interacting components, and they are embedded within larger systems. Components of a larger system act as the context for all lowerlevel systems. Their effects can be pervasive, and thus be taken for granted or regarded as unchangeable. We identify four contextual factors that affect efforts to strengthen HIS: hierarchical roles, aid funding, corruption, and competing priorities. We provide examples of each as experienced by those working to strengthen HIS in LMICs. Each of these contextual factors can seriously diminish the effectiveness of HIS strengthening efforts and their long-term sustainability. We propose research questions about each that would enable those engaged in HIS strengthening to work effectively and sustainably.
\end{abstract}

\section{ARTICLE HISTORY}

Received 1 May 2015

Accepted 4 October 2016

\section{KEYWORDS}

Health information systems; systems perspective; contextual factors; research agenda

\section{Background}

At the turn of the century, several major efforts were launched to combat AIDS and other major epidemics affecting low- and middle-income countries (LMICs). They included the Bill \& Melinda Gates Foundation in 2000, the multilateral Global Fund to Fight AIDS, Tuberculosis and Malaria (the Global Fund) in 2002, and the U.S. bilateral President's Emergency Plan for AIDS Relief (PEPFAR) in 2003. Donors typically want to know that their funds have achieved their intended effect. For example, with global diseases, the effects could include a decrease in overall cases, or decreases in certain high-priority populations. To achieve such change, donors might also wish to see that the systems for implementing necessary health measures, such as distribution of antiretroviral drugs and malaria bed nets, are functioning as needed in the recipient countries. Monitoring health outcomes and health programme systems requires another system in turn: a health information system (HIS) that collects data, analyses them, and presents them to the policy-makers who are aiming to bring about lower rates of disease through improved 
health systems. Health is just one sector of a government. Others, such as the census bureau, agriculture, education, and commerce also receive development funds. A complete HIS includes data from these other sectors, such as vital events and population censuses. Data are needed, then, for the dual purposes of achieving impact and for accountability to donors.

To these ends, the Global Fund and PEPFAR strategies included efforts to strengthen HIS (Thomas et al., 2014). The U.S. government's effort to enhance the capacity of countries to monitor and evaluate health programmes included the creation of the MEASURE Evaluation project in 2003, which is funded in part by PEPFAR, and remains its flagship HIS programme. From 2005 to 2013, the World Health Organization contributed to building HIS through the creation of the Health Metrics Network (HMN). The mission of HMN, in part, was to 'to increase the availability of information for decisions to improve health outcomes in countries' (see Box 1). Presently, WHO and other organisations, such as the Bill and Melinda Gates Foundation and the Global Fund, are advocating for HIS strengthening to enable monitoring of progress towards the international sustainable development goals through a programme called Monitoring and Accountability for Results in Health (MA4Health) and the Health Data Collaborative.

Box 1.

The health information system provides the underpinnings for decision-making and has four key functions: data generation, compilation, analysis and synthesis, and communication and use. The health information system collects data from the health sector and other relevant sectors, analyses the data and ensures their overall quality, relevance and timeliness, and converts data into information for health-related decision-making. (Health Metrics Network, as cited in World Health Organization [WHO], 2010)

In his book, Seeing like a state, Scott argues that many systems created by states have failed to achieve their intended ends because they have oversimplified complex situations and failed to notice or address important factors (1998). He argues that states seek to enhance their control over their domains by simplifying complexity through standards of measurement. Similarly, Ramalingam (2013) argues in his book, Aid on the edge of chaos, that multi- and bilateral aid efforts are dominated by linear, mechanistic approaches. In doing so, they fail to address systems, which are characterised by multiple factors all affecting each other. A systems approach, says Ramalingam, is needed for effectiveness and sustainability of project products. Sustainability can be understood as their use, maintenance, and further improvement after donor funding ends.

USAID recognises the importance of systems thinking in their document, Local systems: A framework for supporting sustained development (2013). They define a local system as 'those interconnected sets of actors - governments, civil society, the private sector, universities, individual citizens and others - that jointly produce a particular development outcome'. Systems thinking encompasses the ability to see system components and how they relate to each other, and to engage with the whole system effectively. The principles for working with systems identified in USAID's document include flexibility, and monitoring and evaluating sustainability. Monitoring complex systems is addressed more fully in the USAID document, Complexity-aware monitoring (2014).

Systems are typically conceived as consisting of eight characteristics. They are: selforganising constantly changing, tightly linked, governed by feedback, non-linear, history-dependent, counter-intuitive, and resistant to [intentional] change (Meadows, 
Richardson, \& Bruckmann, 1982; Sterman, 2006). All systems are contained or nested within larger systems. The elements of a system description depend on the system level being described.

An application of systems thinking to the health sector is provided by de Savigny and Adam (2009) in a conceptual diagram of the components of a health system and how they relate to each other (Figure 1). The mention of accountability in the figure may hint at a higher-level system that takes into account broader societal attitudes towards transparency. And the mention of case management may hint at lower-level systems to manage patients within a health facility. The detail level of Figure 1 and lower levels are the ones most commonly described, perhaps because they incorporate elements that lend themselves to measurement and to intervention; in contrast to, say, societal attitudes towards transparency. Yet, broad contextual factors such as societal attitudes can influence virtually every aspect of a lower-level system. Because the influence of contextual factors is pervasive, those working with the system may feel that they are out of their control and must be taken as a given.

We believe an understanding of a contextual factor is essential to achieving sustainable change in any system embedded within the broader context. Our purpose here is to identify several important contextual factors affecting HIS strengthening, and to propose a research agenda that might enable such strengthening efforts to be more effective and sustainable. Our observations will be relevant to donor agencies that set priorities and allocate resources for HIS strengthening, and researchers who can study the contextual factors we identify.

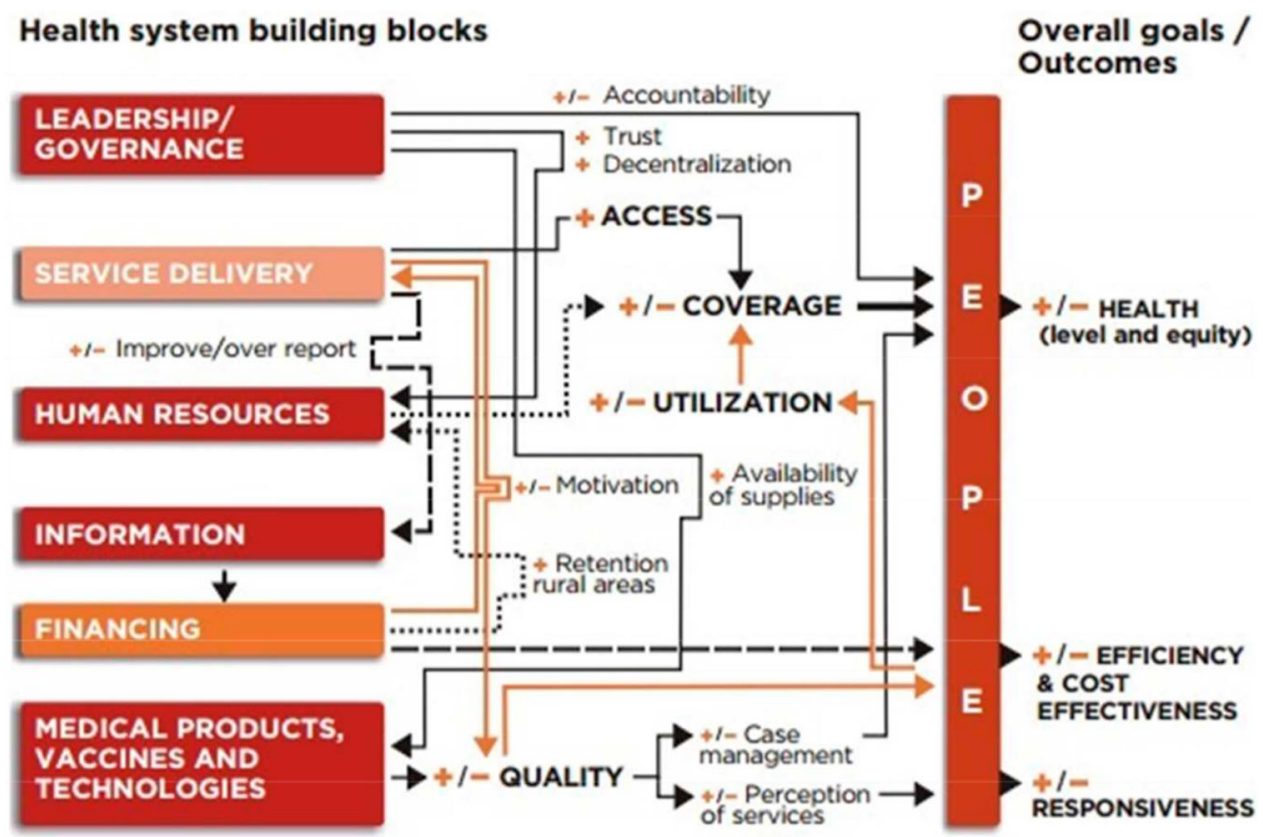

Figure 1. Example of interrelationships between the components of a health system (de Savigny \& Adam, 2009). 


\section{Contextual factors}

Many of the technical staff of the MEASURE Evaluation project have decades of experience strengthening HIS in LMICs, with MEASURE Evaluation as well as with other projects. We asked the project staff (more than 100 in number) for examples of contextual factors that have frustrated or facilitated their work. Four factors were identified: hierarchical roles, aid funding, corruption, and competing priorities of the host government. These factors are not fully independent of each other; they may act alone or in combination. However, there is no clear evidence of the conditions affecting their influence on each other. For each of them, we provide examples from experience (without mention of country names), insights on the factor from recent literature, and questions for research.

\section{Hierarchical roles}

Example 1: In a leadership development activity, middle management teams created plans and were prepared to implement them. However, their supervisor refused to let them do so, noting that it was not their role to conceive plans much less implement them.

Example 2: While building leadership for data demand and use, one group identified a need in their country for an HIS technical working group. The Minister of Health publicly approved the creation of such a group but did not appoint members, effectively disapproving it. In the absence of action at the top, the need for a technical coordinating group remains unmet.

Example 3: A set of health providers who had received training in the use of data for decision-making were failing to implement their data use skills. They reported that they had no professional incentive to do so. They were not required by their supervisors to use data for decision-making, and their performance evaluations did not include data use. They were required to collect the data, however, so they simply passed it up the line without using it themselves.

Each of these is an example in which the location of actors on a hierarchical ladder determined their personal investment in the system, their interest in taking action to improve the system, and the success of their actions. In one instance, the lower-level workers did only what they were required to do, and gave no evidence of being invested in the system's larger purpose. In the other examples, the engagement demonstrated by lower-level workers was discouraged by their superiors.

In his study of work-related values among employees in IBM offices in 72 countries, Hofstede described this adherence to hierarchy as power distance (1980, 2001). Various researchers have applied Hofstede's framework to the implementation of information systems (De Man, 2003; Erik De Man \& Van den Toorn, 2002; Grover, Segars, \& Durand, 1994; Shore \& Venkatachalam, 1996). The GLOBE study of organisational leadership in 62 societies in the 1990s also identified power distance as a cultural dimension (House, Hanges, Javidan, Dorfman, \& Gupta, 2004). Cultural anthropologist, Mary Douglas places hierarchical social relationships within a group/grid framework. Hierarchical social relations exist where group membership is strong and where behaviours are highly regulated (high-grid) (Thompson, Ellis, \& Wildavsky, 1990). These might coexist, for example, in a group delineated by strong ethnic identity and strong traditions regulating interactions within the ethnic group. Hierarchical roles might be considered 
an aspect of culture, which has been defined in one instance as 'learned patterns of thought and behavior characteristic of a social group' (Brown, 1997, p. 119). In cultures with hierarchical relationships, the respective roles of workers and their supervisors are clearly delineated and adhered to. Decision-making is 'top down' or directed from the upper reaches of the hierarchy, and implemented dutifully by subordinates. In a low hierarchy culture, management is sometimes described as 'flat' or a team structure. Input from subordinates is sought in making decisions, and subordinates are encouraged to catch and correct errors or address unforeseen contingencies, in some cases without waiting for input from superiors.

A given country can demonstrate elements of both high and low hierarchy. For example, in the U.S., democratic processes in civic and business settings are consistent with low hierarchy, while a high degree of hierarchy is evident in the military, which often depends on quick, authoritative decision-making. Overall, however, the U.S. scored as a relatively low power distance country in the IBM study (Hofstede, 2001), as did most Western countries. LMICs, in contrast, typically scored with a high power distance.

In a high hierarchy setting, workers may be accustomed to making decisions on the basis of those handed down to them from higher echelons, or according to the authority given to them. They may exercise any authority they have autocratically, without input from others, or after carefully considering data and others' opinions. Depending on the patterns set by the superior, subordinates may filter information and provide only that which pleases the superior and supports his or her preconceptions; or provide unfiltered information that enables the superior to make data-informed decisions. In the former situation, data viewed as objective by a person from a low hierarchy culture can be viewed as superfluous or even subversive by a person from a high hierarchy setting. In such an instance, then, the decision-maker in the high hierarchy setting may resist the implementation of evidence-based decision-making.

Research questions to elucidate the role of hierarchical structures on HIS strengthening might include: the relevant manifestations of hierarchical roles in an HIS and how are they best measured; the instances in which hierarchical roles facilitate or frustrate HIS strengthening; whether institutional practices based in hierarchical roles can be changed to facilitate a high-functioning HIS; and other instances in which aspects of culture facilitate or frustrate HIS strengthening (Table 1).

\section{Aid funding}

Example 1: In the creation of a national HIV/AIDS database in one country, donor funding for data collection and reporting was going to one ministry, but the responsibility for the effort was placed on another. In the absence of funding, the second ministry showed no interest in taking on the responsibilities.

Example 2: Donor-funded community surveys were conducted country-wide to identify needed improvements in health service delivery. Much benefit was realised, but there was little evidence that the communities would continue the process on their own when the external funding ends.

Example 3: In some countries, outside funding is not provided for HIS strengthening, but an outside expert is temporarily stationed in the Ministry of Health (MOH) as a 
Table 1. Potential research questions pertaining to four contextual factors affecting health information systems in low-to-middle-income countries.

\section{Hierarchical structures}

- What are the relevant manifestations of power distance in a health information system (HIS) and how are they best measured?

- In what instances do hierarchical roles facilitate or frustrate HIS strengthening?

- Can institutional practices based in hierarchical roles be changed to facilitate a high-functioning HIS?

- In what instances do aspects of culture facilitate or frustrate HIS strengthening?

\section{Aid funding}

- How is aid funding best measured?

- How is local initiative or investment best measured?

- Which aid funding practices discourage or encourage local initiative?

- What practices enable aid funding to be coordinated among donors and sectors?

\section{Corruption}

- Which HIS practices facilitate transparency in a way that is valued by both the donors and local users?

- Does transparency engender trust in data, data use, and further investment in the HIS?

\section{Competing priorities}

- What inputs (e.g. capacity building among those collecting or using data) achieve HIS strengthening?

- Does a strong HIS lead to better performing delivery of health services and health outcomes?

- Is population health associated with non-health outcomes, such as worker productivity?

resource for planning that is initiated by the Ministry. In several instances, the ministries continued to apply the process after the departure of the technical expert. However, when donors offered large sums to support their favoured strategies, the national health planning process began to crumble.

In these examples, outside funding that aims to make improvements has the unintended consequence of disempowering local implementers. In two instances, the willingness to act is tied to money. Where there is none to be had, there is either no interest in participating, or a successful effort loses steam. The perception that donor money disempowers local efforts is reinforced in the third example, where progress was made when the donor provided only a temporary advisor, but was lost when external donor agendas were imposed.

In Dead aid, Zambian-born economist Dambisa Moyo argues that international aid hinders rather than helps development (2009). She cites examples of advances in countries that have actively minimised international aid (e.g. Botswana), and of stalled progress or even regression in those accepting large amounts of aid (e.g. Zimbabwe). She suggests that LMICs should be weaned off of international aid so they will begin to act of their own accord in establishing their needed markets, policies, and programmes.

Others argue for aid reform, not elimination. In White man's burden, William Easterly describes two types of people guiding development: planners and searchers (2006). Planners, he says, 'apply global blueprints; searchers adapt to local problems. Planners at the top lack knowledge of the bottom; searchers find out what the reality is at the bottom' (p. 6). Societies are complex and unique, says Easterly; they cannot be programmed into success. Rather, they must grow bit by bit through trial and error. The example above in which an expert was placed temporarily in the $\mathrm{MOH}$ as a resource is a searcher approach. The expert was there to see what the staff in the $\mathrm{MOH}$ experienced, and to be available to respond to their questions or requests for assistance. Similarly, Ramalingam (2013) describes development as a complex adaptive system in which local practical knowledge can lead to effective and sustainable solutions. This stands in contrast to 
solutions prescribed from afar and implemented similarly and mechanistically, regardless of the location.

The first example underscores how aid funding is often sector-specific. However, an HIS incorporates data from several sectors. If they are not coordinated, their data systems will not be interoperable with each other, thus decreasing the overall utility and power of the HIS. Categorical aid funding, and accountability to particular donors for particular sectors, can lead to this unintended outcome.

Research questions addressing the contextual influences of aid funding include: how aid funding is best operationalised and measured?; how local initiative or investment is best measured?; which aid funding practices discourage or encourage local initiative?; and what practices enable aid funding to be coordinated among donors and sectors?

\section{Corruption}

Example 1: One MEASURE Evaluation staff member reported that the worst corruption he had seen was during relief following an earthquake. The military kept funds that were intended for rebuilding houses and community infrastructure.

Example 2: Over a number of years, a large number of information and communications technology (ICT) resources for HIS, including computers, were provided to one country from a variety of sources, including the World Bank, the Global Fund, and the multilateral Roll Back Malaria initiative. However, in a country-wide ICT inventory assessment done later, much of the equipment could not be located.

The disappearance or diversion of resources to personal accounts or pet projects has a long history in virtually every country. Corruption in international aid may stand out because the amounts are large, they are often from taxpayers' contributions and are thus followed closely by the general population, and they are to countries that often lack transparency in the distribution of funds. The lack of transparency is in the levels of society that receive and manage international donations, which are typically the upper echelons of a hierarchical society. Transparency International has developed a Corruption Perception Index (CPI) as a measure of transparency. Hofstede found that where power distance was greater, corruption as measured by the CPI was also greater (2001, p. 112). Those in power who are gaining from corruption have an interest in avoiding transparency, and thus perhaps data systems that promote objectivity and transparency.

In their analysis of the relationship between foreign aid and corruption, Alesina and Weder (1999) found that countries on the upper end of the corruption scale received more aid money. Moyo took this finding further, asserting that aid actually fosters corruption (2009, p. 52). Ghanaian-born economist George Ayittey refers to countries in which the elite are steeped in corruption as 'Vampire States'. In these states, he explains, the elites suck the lifeblood out of the country (Ayittey, 2005, p. 239).

But the picture in many LMICs is changing. In The great surge, Steven Radelet describes how the rate of extreme poverty has plummeted in LMICs since the 1980s (2015). He attributes this, in part, to the decline of autocratic governments and corresponding increases in democratic ones. He notes that in 1983, 17 LMICs were democracies; by 2013 the number had more than tripled to 56 (Ramalingam, 2013, p. 6). With the association between power distance and corruption mentioned above, a shift from autocracy to democracy could be accompanied by a decline in corruption. 
As mentioned above, MA4Health is one example of an initiative to ensure that funds achieve their intended results. Another example is a PEPFAR initiative called Data for Accountability, Transparency, and Impact (DATIM), which collects data from all facilities receiving PEPFAR funds. The data are then used by American Missions to determine where the country's needs are greatest and where the most progress has been realised. At present, DATIM is designed to inform the donor, the U.S. government. Once in place, the hope is to translate lessons learned in DATIM to the host government HIS.

Health is just one sector of a government. Others, such as the census bureau, agriculture, education, and commerce also receive development funds. A complete HIS includes data from these other sectors, such as vital events and population censuses, and data collected in targeted studies. Each donor will be interested in accountability for their funds invested in each sector. When many donors want information to guide their programmes, the number and types of information sought can mushroom. Those working in a clinic or community-based organisation may spend more and more time gathering data, and less time delivering health services to clients. The benefits of data need to be weighed against the burden of collecting them, and the resentment that may result in resistance to data collection (Thomas, Silvestre, Salentine, Reynolds, \& Smith, 2016).

Health information systems implemented to provide donors accountability for their funds address the contextual challenges of corruption, but do so at the risk of the unintended consequences of aid funding, previously mentioned. The host governments are involved little, except in providing data, and have little invested in the system, except their interest in continuing to qualify for donor funds. As described above, this raises concerns about the quality of implementation and long-term sustainability. If the host country does not have a sense of ownership of the system, they will not have the incentive to maintain it and use it.

Questions about accountability and transparency in HIS strengthening that would benefit from research include: the HIS practices that facilitate transparency in way that is valued by both the donors and local users; and whether transparency engenders trust in data, data use, and further investment in the HIS.

\section{Competing priorities}

Example 1: In one of the countries affected by the recent Ebola epidemic, competition for government funds among the various government sectors was so intense that when the $\mathrm{MOH}$ first went to the Parliament for additional funding to fight Ebola, those representing the non-health sectors argued against the requested resources, suggesting that the report of an epidemic was only a ruse for the $\mathrm{MOH}$ to get more than their share of funds.

Example 2: In a statement about country priorities, a donor wrote,

The [country] government has placed a priority on economic growth and infrastructure to the detriment of investments in health. Competing priorities in the health sector have also contributed to governmental investment and support that is currently inadequate for HIV control. Delays in adoption of policies known to better enrollment and retention on HIV treatment and care have impeded progress.

Example 3: In a recent political campaign, one of the parties argued that the incumbent was more interested in investing in projects that enhance the country's image among 
foreigners, such as luxury hotels, than it was in taking care of basic country needs, such as schools, roads, and the health system. In contrast to the corrupt diversion of resources, these are examples of funds used for purposes other than HIS through processes that are relatively transparent, albeit controversial and political. The arguments presented are the mainstay of politics: competing views on the allocation of scarce resource for the benefit of the population.

Although the political environment serves as a backdrop to HIS strengthening, it is often changing, particularly in democracies where the parties in power change, along with the political appointments reflecting the party's priorities. Where change is possible, engagement in bringing it about is more common. For this reason, politics and changing priorities often remain present in the minds of government employees and the general population, perhaps more so than culture, aid funding, and corruption.

Where there are competing ideas of where resources should be invested, data-driven evidence of effectiveness ought to play an important role. Trustworthy data are important, but they are seldom the sole consideration in policy-making. Other considerations include competing data-driven arguments, payback for political favours, and appeasement of the population to enhance one's chances for re-election. If policy-makers were to value the HIS, they would likely demonstrate it by funding the resources needed to ensure its quality and relevance. But one must overcome the vicious cycle in which the HIS must first be valued enough to attract the resources needed to produce useful data.

In a country where the HIS is not a priority because it is not valued, the evidence of its utility and value will likely come from countries where the HIS is valued and data on its performance and effects are available (Thomas et al., 2014). Research that could demonstrate the value of an HIS might address evidence of the types of input that achieve HIS strengthening; evidence that a strong HIS leads to better performing delivery of health services and health outcomes; and evidence of the effects of a healthy population on nonhealth outcomes, such as worker productivity.

\section{Summary and conclusions}

We have identified four contextual factors that people implementing HIS strengthening reported to have affected the success of their work. The factors are not mutually exclusive; each can affect or coincide with another. And the list is most certainly not exhaustive. For example, a contextual factor not addressed here is the country economy. A country with a relatively low gross domestic product, even compared to other LMICs, will have trouble finding the resources, including trained personnel, to participate in HIS strengthening projects - even while funded by an outside donor. The relevant contextual factors will be case dependent.

We have not fully explored here how any of the contextual factors described could, in some circumstances, work to the benefit of HIS strengthening. For example, in a hierarchical culture, a high-level decision-maker could insist on data-informed decisions and thus designate resources to establish a system for collecting reliable data.

The contextual factors we address reflect a systems perspective to strengthening an HIS. As systems are nested within each other, these contextual factors influence each of the systems nested within. They can determine whether strengthening occurs and whether the performance of the HIS remains strong after donor funding is discontinued. 
Because they can set the context for an entire country, they are at risk of not being seen at all, or being regarded as unchangeable, and thus not worth studying. Factors that are easily measured and easily changed are more likely to be the subject of study. We believe, however, that useful information on the questions we have listed can be obtained within a few years, a common study timeframe. In doing so, researchers would need to identify how best to include contextual factors in systems diagrams and how to measure and monitor the factors. Because the research questions pertain to complex adaptive systems, the research methods are not likely to be those applied to more mechanistic, linear processes, such as a randomised controlled trial (Thomas, Curtis, \& Smith, 2011; USAID, 2013). Nonetheless, the methods can be rigorous and reliable, and the findings informative and actionable. To avoid such research is to risk pouring resources into programmes that do not achieve their goals and will not be sustained after donor funding has ended.

\section{Acknowledgements}

The author thanks the members of the MEASURE Evaluation project staff who contributed examples of contextual factors affecting their work. The views expressed in this article are those of the authors and not necessarily those of USAID. USAID had no role in the writing or approval of the manuscript. The author conceived of the article, drafted the manuscript and revised it critically for important intellectual content.

\section{Disclosure statement}

No potential conflict of interest was reported by the author.

\section{Funding}

Preparation of this article was supported by the United States Agency for International Development MEASURE Evaluation, Phase IV Award (AID-OAA-L-14-00004).

\section{ORCID}

James C. Thomas (D) http://orcid.org/0000-0002-2225-2052

\section{References}

Alesina, A., \& Weder, B. (1999). Do corrupt governments receive less foreign Aid? Cambridge, MA: National Bureau of Economic Research. Retrieved from http://www.nber.org/papers/w7108

Ayittey, G. (2005). Africa unchained: The blueprint for Africa's future. New York, NY: Palgrave Macmillan.

Brown, P. (1997). Culture and the global resurgence of malaria. In M. C. Inhorn \& P. Brown (Eds.), The anthropology of infectious disease: International health perspectives (pp. 119-144). New York, NY: Routledge.

De Man, W. H. E. (2003). Cultural and institutional conditions for using geographic information: Access and participation. Journal of the Urban and Regional Information Systems Association, 15 (APA2), 29-33.

Easterly, W. (2006). The White man's burden: Why the West's efforts to aid the rest have done so much ill and so little good. New York, NY: The Penguin Press. 
Erik de Man, W. H., \& Van den Toorn, W. (2002). Culture and the adoption and use of GIS within organisations. International Journal of Applied Earth Observation and Geoinformation, 4, 51-63.

Grover, V., Segars, A. H., \& Durand, D. (1994). Organizational practice, information resource deployment and systems success: A cross-cultural survey. The Journal of Strategic Information Systems, 3(2), 85-106.

Hofstede, G. (1980). Culture's consequences: International differences in work-related values. Thousand Oaks, CA: SAGE Publication.

Hofstede, G. (2001). Culture's consequences: Comparing values, behaviors, institutions, and organizations across nations (2nd ed.). Thousand Oaks, CA: SAGE Publication.

House, R. J., Hanges, P. J., Javidan, M., Dorfman, P. W., \& Gupta, V. (2004). Culture, leadership, and organizations: The GLOBE study of 62 societies. Thousand Oaks, CA: SAGE Publication.

Meadows, D., Richardson, J., \& Bruckmann, G. (1982). Groping in the dark: The first decade of global modelling. New York, NY: Wiley.

Moyo, D. (2009). Dead aid: Why aid is not working and How there is a better Way for Africa. New York, NY: Farrar, Straus and Giroux.

Radelet, S. (2015). The great surge: The ascent of the developing world. New York, NY: Simon and Schuster.

Ramalingam, B. (2013). Aid on the edge of chaos. Oxford: Oxford University Press.

de Savigny, D., \& Adam, T. (2009). Systems thinking for health systems strengthening. Alliance for Health Policy and Systems Research, WHO Retrieved from http://apps.who.int/iris/bitstream/ 10665/44204/1/9789241563895_eng.pdf.

Scott, J. (1998). Seeing like a state: How certain schemes to improve the human condition have failed. New Haven, CT: Yale University Press.

Shore, B., \& Venkatachalam, A. R., 1996. Role of national culture in the transfer of information technology. The Journal of Strategic Information Systems 5, 19-35.

Sterman, J. D. (2006). Learning from evidence in a complex world. American Journal of Public Health, 96, 505-514.

Thomas, J. C., Curtis, S., \& Smith, J. (2011). The broader context of implementation science [letter]. JAIDS, 58, e19-e21.

Thomas, J. C., Hardee, K., Parks, A., Boone, D., Brown, W., Pacquée-Margolis, S., \& Tran Ba Huy, R. (2014). Global action networks in health: A case study of data quality. Global Health Governance, VII, 80-95.

Thomas, J. C., Silvestre, E., Salentine, S., Reynolds, H., \& Smith, J. (in press). The iceberg below the indicators: What systems are essential to achieving the sustainable development goals and what will it take to marshal them? Health Policy and Planning.

Thompson, M., Ellis, R., \& Wildavsky, A. (1990). Cultural theory. Boulder, CO: Westview Press.

USAID. (2013, December). Complexity-aware monitoring, Version 2.0. Retrieved from http:// usaidlearninglab.org/sites/default/files/resource/files/Complexity\%20Aware\%20Monitoring\% 202013-12-11\%20FINAL.pdf

USAID. (2014). Local systems: A framework for supporting sustained development. Washington, DC. Retrieved from http://www.usaid.gov/sites/default/files/documents/1870/LocalSystemsFrame work.pdf

World Health Organization. (2010). Monitoring the building blocks of health systems: A handbook of indicators and their measurement strategies. Geneva: Author. Retrieved from http://www.who. int/healthinfo/systems/WHO_MBHSS_2010_full_web.pdf 